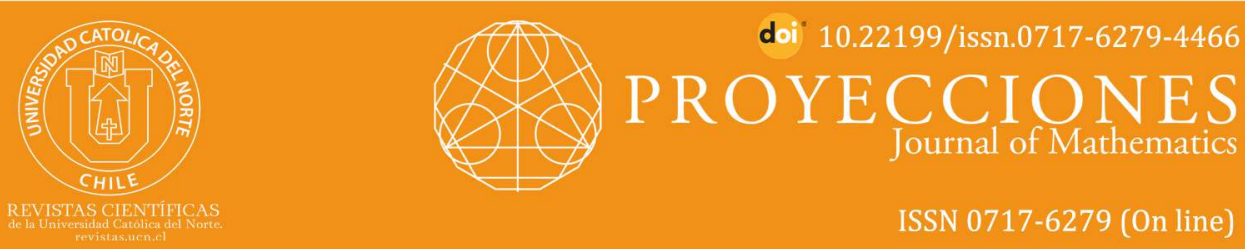

\title{
$\lambda$-quasi Cauchy sequence of fuzzy numbers
}

\author{
Achyutananda Baruah ${ }^{1}$

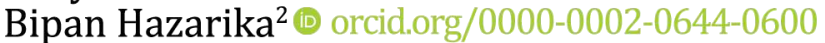

${ }^{1}$ North Gauhati College, Dept. of Mathematics, Guwahati, AS, India.

achyutanandabaruah@gmail.com

${ }^{2}$ Gauhati University, Dept. of Mathematics, Guwahati, AS, India.

घbh_rgu@yahoo.co.in

\section{Abstract:}

In this paper we introduce the $\lambda$-quasi Cauchy sequence of fuzzy numbers. We obtain the relation between strongly $\lambda$-quasi Cauchy convergence and statistically $\lambda$-quasi Cauchy convergence for fuzzy numbers.

Keywords: Fuzzy number; Quasi Cauchy sequence; Statistical convergence; Solidness; Symmetricity and convergence free.

MSC (2020): 03E72, 40C05, 40J05, 40G15, 46A45, 46S40.

\section{Cite this article as (IEEE citation style):}

A. Baruah and B. Hazarika, " $\lambda$-quasi Cauchy sequence of fuzzy numbers", Proyecciones (Antofagasta, On line), vol. 40, no. 4, pp. 981-988, 2021, doi: 10.22199/issn.0717-6279-4466

Article copyright: (C) 2021 Achyutananda Baruah and Bipan Hazarika. This is an open access article distributed under the terms of the Creative Commons License, which permits unrestricted use and distribution provided the original author and source are credited. 


\section{Introduction}

Fuzzy set was first suggested by Zadeh [24]. Bounded and convergent sequences of fuzzy numbers were studied by Matloka [17], where it is shown that every convergent sequence is bounded. Later on different classes of sequences of fuzzy numbers have been studied by Ganie and Sheikh [2] and many others. The idea of statistical convergence for single sequences was introduced by Fast [10] in 1951. Schoenberg [15], studied the statistical convergence as a summability method and listed some of elementary properties of statistical convergence. Both of these authors noted that if bounded sequence is statistically convergent, then it is Cesàro summable. Mursaleen [16] defined and studied $\lambda$-statistically convergent sequences (also see $[1,14]$ ). The notion was further investigated and different properties in the field of summability theory has been investigated by Tripathy [18], Tripathy and Sen [19], Tripathy and Esi [20], Tripathy and Das [23], and many others.

\section{Preliminaries}

A fuzzy real number $X$ is fuzzy set on $\mathbf{R}$ and is a mapping $X: \mathbf{R} \rightarrow$ $I(=[0,1])$ associating each real number $t$ with its grade membership $X(t)$. For the definition of normal, upper-semi continuous and convex fuzzy real numbers denoted by $\mathbf{R}(I)$ (one may refer Tripathy and Baruah [21]).

Throughout the article $w^{F}, c^{F}, c_{0}^{F}, \ell_{\infty}^{F}$ denote the classes of all, convergent, null, bounded sequences of fuzzy real numbers, respectively.

We denote $D$, the set of all the closed and bounded intervals on the real line R. For any $X, Y \in D$ we define

$$
d(X, Y)=\max \left(\left|a_{1}-a_{2}\right|,\left|b_{1}-b_{2}\right|\right) \text { where } X=\left[a_{1}, b_{1}\right], Y=\left[a_{2}, b_{2}\right] .
$$

Clearly $(D, d)$ is a complete metric space.

Let $\bar{d}: \mathbf{R}(I) \times \mathbf{R}(I) \rightarrow \mathbf{R}$ be defined by

$$
\bar{d}(X, Y)=\sup _{0 \leq \alpha \leq 1} d\left(X^{\alpha}, Y^{\alpha}\right) .
$$

Then $\bar{d}$ is a metric on $\mathbf{R}(I)$ (One may refer to Matloka [17]). The additive identity and the multiplicative identity in $\mathbf{R}(I)$ are denoted by $\overline{0}$ and $\overline{1}$, respectively.

A fuzzy real valued sequence $\left(X_{k}\right)$ is said to be convergent to a fuzzy real number $X_{0}$, if for every given $\varepsilon>0$, there exists $n_{0} \in \mathbf{N}$ such that $\bar{d}\left(X_{k}, X_{0}\right)<\varepsilon$ for all $k \geq n_{0}$. 
A fuzzy real valued sequence $\left(X_{k}\right)$ is said to be Cauchy sequence if for a given $\varepsilon>0$, there exists $n_{0} \in \mathbf{N}$ such that $\bar{d}\left(X_{k}, X_{n}\right)<\varepsilon$ for all $k, n \geq n_{0}$.

For $X=\left(X_{k}\right)$, a given sequence $S(X)$ denotes the set of all permutation of the elements of $\left(X_{k}\right)$ that is $S(X)=\left(X_{\pi(k)}\right)$.

A sequence space $E^{F}$ is said to be solid if $\left(Y_{k}\right) \in E^{F}$ whenever $\left|Y_{k}\right| \leq$ $\left|X_{k}\right|$ for all $k \in \mathbf{N}$ and $\left(X_{k}\right) \in E^{F}$.

A sequence space $E^{F}$ is said to be sequence algebra if $\left(X_{k} Y_{k}\right) \in E^{F}$ whenever $\left(X_{k}\right)$ and $\left(Y_{k}\right)$ in $E^{F}$.

A sequence space $E^{F}$ is said to be convergence free if $\left(Y_{k}\right) \in E^{F}$ whenever $\left(X_{k}\right) \in E^{F}$ and $X_{k}=0$ implies $Y_{k}=0$.

Definition 2.1. A sequence $X=\left(X_{k}\right)$ of fuzzy numbers is said to be convergent to the fuzzy number $X_{0}$ if for each $\varepsilon>0$ there exists a positive integer $k_{0}$ such that $\bar{d}\left(X_{k}, X_{0}\right)<\varepsilon$ for all $k \geq k_{0}$ and we denote it by $\lim _{k} X_{k}=X_{0}$. Thus, $\lim _{k} X_{k}=X_{0} \Longleftrightarrow \lim _{l} X_{k_{l}}=X_{0_{l}}$ and $\lim _{r} X_{k_{r}}=X_{0_{r}}$.

In the following way Burton and Coleman [3] defined quasi Cauchy sequence.

A sequence $X=\left(X_{k}\right)$ of points in $\mathbf{R}$ is called quasi Cauchy if $\Delta x_{k}$ is a null sequence, where $\Delta x_{k}=x_{k}-x_{k+1}$.

For quasi-Cauchy sequences one can see $[4,5,6,8,7,9]$. We refer $([11,12,13,22]$ and references therein) for difference sequence of fuzzy numbers.

In section 3 , we introduce and discuss the concept of strongly $\lambda$-quasi Cauchy convergence and statistically $\lambda$-quasi Cauchy convergence of fuzzy numbers.

\section{3. $\lambda$-quasi Cauchy convergence of fuzzy numbers}

Definition 3.1. Let $\lambda=\left\{\lambda_{n}\right\}$ be a non-decreasing sequence of positive numbers such that $\lambda_{n+1} \leq \lambda_{n}+1, \lambda_{1}=1, \lambda_{n} \rightarrow \infty$ as $n \rightarrow \infty$ and $I_{n}=$ $\left[n-\lambda_{n}+1, n\right]$. A sequence $X=\left(X_{k}\right)$ of fuzzy numbers is said to be strongly $\lambda$-quasi Cauchy summable if there is an fuzzy number $X_{0}$ such that

$$
\lim _{n} \frac{1}{\lambda_{n}} \sum_{k \in I_{n}} \bar{d}\left(\Delta X_{k}, X_{0}\right)=0
$$

In which case we say that the sequence $X=\left(X_{k}\right)$ of fuzzy numbers is said to be strongly $\lambda$-quasi Cauchy summable to fuzzy number $X_{0}$ and it is denoted by $w^{F}(\lambda)$. 
Definition 3.2. A sequence $X=\left(X_{k}\right)$ of fuzzy numbers is said to be statistically $\lambda$-quasi Cauchy convergent to fuzzy number $X_{0}$ if for every $\varepsilon>0$

$$
\lim _{k} \frac{1}{\lambda_{n}}\left|\left\{k \in I_{n}: \bar{d}\left(\Delta X_{k}, X_{0}\right) \geq \varepsilon\right\}\right|=0 .
$$

In this case we write $S_{\lambda}-\lim \Delta X_{k}=X_{0}$.

Theorem 3.3. Let $X=\left(X_{k}\right)$ and $Y=\left\{Y_{k}\right\}$ be two sequences of fuzzy numbers.

(i) If $S_{\lambda}-\lim \Delta X_{k}=X_{0}$ and $\alpha \in \mathbf{R}$, then $S_{\lambda}-\lim \alpha \Delta X_{k}=\alpha X_{0}$.

(ii) If $S_{\lambda}-\lim \Delta X_{k}=X_{0}$ and $S_{\lambda}-\lim \Delta Y_{k}=Y_{0}$. Then $S_{\lambda}-\lim \left(\Delta X_{k}+\right.$ $\left.\Delta Y_{k}\right)=X_{0}+Y_{0}$

Proof. (i) Let $\alpha \in \mathbf{R}$. We have $\bar{d}\left(\alpha \Delta X_{k}, \alpha X_{0}\right)=|\alpha| \bar{d}\left(\Delta X_{k}, X_{0}\right)$. For a given $\varepsilon>0$ we have

$$
\frac{1}{\lambda_{n}}\left|\left\{k \in I_{n}: \bar{d}\left(\alpha \Delta X_{k}, \alpha X_{0}\right) \geq \varepsilon\right\}\right| \leq \frac{1}{\lambda_{n}}\left|\left\{k \in I_{n}: \bar{d}\left(\Delta X_{k}, X_{0}\right) \geq \frac{\varepsilon}{|\alpha|}\right\}\right| .
$$

Hence $S_{\lambda}-\lim \alpha \Delta X_{k}=\alpha X_{0}$.

(ii) Suppose $S_{\lambda}-\lim \Delta X_{k}=X_{0}$ and $S_{\lambda}-\lim \Delta Y_{k}=Y_{0}$. We have

$$
\bar{d}\left(\Delta X_{k}+\Delta Y_{k}, X_{0}+Y_{0}\right) \leq \bar{d}\left(\Delta X_{k}, X_{0}\right)+\bar{d}\left(\Delta Y_{k}, Y_{0}\right) .
$$

Therefore, for given $\varepsilon>0$ we have

$$
\begin{aligned}
& \frac{1}{\lambda_{n}}\left|\left\{k \in I_{n}: \bar{d}\left(\Delta X_{k}+\Delta Y_{k}, X_{0}+Y_{0}\right) \geq \varepsilon\right\}\right| \mid \\
\leq & \frac{1}{\lambda_{n}}\left|\left\{k \in I_{n}: \bar{d}\left(\Delta X_{k}, X_{0}\right)+\bar{d}\left(\Delta Y_{k}, Y_{0}\right) \geq \varepsilon\right\}\right| \mid \\
\leq & \frac{1}{\lambda_{n}}\left|\left\{k \in I_{n}: \bar{d}\left(\Delta X_{k}, X_{0}\right) \geq \frac{\varepsilon}{2}\right\}\right|+\frac{1}{\lambda_{n}}\left|\left\{k \in I_{n}: \bar{d}\left(\Delta Y_{k}, Y_{0}\right) \geq \frac{\varepsilon}{2}\right\}\right| .
\end{aligned}
$$

Hence $S_{\lambda}-\lim \left(\Delta X_{k}+\Delta Y_{k}\right)=X_{0}+Y_{0}$.

In the following theorems, we exhibit some connections between strongly $\lambda$-quasi Cauchy summable and statistically $\lambda$-quasi Cauchy convergence of sequences of fuzzy numbers.

Theorem 3.4. If a fuzzy sequence $X=\left(X_{k}\right)$ is strongly $\lambda$-quasi Cauchy summable to fuzzy number $X_{0}$ then it is statistically $\lambda$-quasi Cauchy convergent to fuzzy number $X_{0}$. 
Proof. Let $\varepsilon>0$, be given. We have the result from the following inequality. $\begin{aligned} \frac{1}{\lambda_{n}} \sum_{k \in I_{n}} \bar{d}\left(\Delta X_{k}, X_{0}\right) & \geq \frac{1}{\lambda_{n}} \sum_{k \in I_{n}, \bar{d}\left(\Delta X_{k}, X_{0}\right) \geq \varepsilon} \bar{d}\left(\Delta X_{k}, X_{0}\right) \\ & \geq \frac{1}{\lambda_{n}}\left|\left\{k \in I_{n}: \bar{d}\left(\Delta Y_{k}, Y_{0}\right) \geq \varepsilon\right\}\right| .\end{aligned}$

Hence the result follows. to fuzzy number $X_{0}$ then it is statistically $\lambda$-quasi Cauchy convergent to fuzzy number $X_{0}$.

Theorem 3.5. If a fuzzy sequence $X=\left(X_{k}\right)$ is statistically quasi Cauchy convergent to fuzzy number $X_{0}$ and $\liminf _{n} \frac{\lambda_{n}}{n}>0$, then it is statistically $\lambda$-quasi Cauchy convergent to $X_{0}$.

Proof. For given $\varepsilon>0$ we have

$$
\left\{k \leq n: \bar{d}\left(\Delta X_{k}, X_{0}\right) \geq \varepsilon\right\} \supset\left\{k \in I_{n}: \bar{d}\left(\Delta X_{k}, X_{0}\right) \geq \varepsilon\right\} .
$$

Therefore

$$
\begin{aligned}
& \frac{1}{n}\left|\left\{k \leq n: \bar{d}\left(\Delta X_{k}, X_{0}\right) \geq \varepsilon\right\}\right| \\
\geq & \frac{1}{n}\left|\left\{k \in I_{n}: \bar{d}\left(\Delta X_{k}, X_{0}\right) \geq \varepsilon\right\}\right| \quad \text { Taking limits as } n \rightarrow \infty \text { and } \\
\geq & \frac{\lambda_{n}}{n} \frac{1}{\lambda_{n}}\left|\left\{k \in I_{n}: \bar{d}\left(\Delta X_{k}, X_{0}\right) \geq \varepsilon\right\}\right| .
\end{aligned}
$$

using the condition $\liminf \frac{\lambda_{n}}{n}>0$, we get $\left(X_{k}\right)$ is statistically $\lambda$-quasi Cauchy convergent to $X_{0}$.

Theorem 3.6. $w^{F}(\lambda)$ is a solid space.

Proof. $\quad$ Let $\left(X_{k}\right)$ and $\left\{Y_{k}\right\}$ be sequences in $w^{F}(\lambda)$ such that $\bar{d}\left(Y_{k}, 0\right) \leq$ $\bar{d}\left(X_{k}, 0\right)$ for each $k \in \mathbf{N}$. Then we get required result from the following inequality

$$
\frac{1}{\lambda_{n}} \sum_{k \in I_{n}} \bar{d}\left(Y_{k}, 0\right) \leq \frac{1}{\lambda_{n}} \sum_{k \in I_{n}} \bar{d}\left(X_{k}, 0\right) .
$$

Theorem 3.7. $w^{F}(\lambda)$ is not a symmetric space.

Proof. For each $k \in \mathbf{N}$ and let us consider the sequences

$$
X=\{A, B, A, B, \ldots\}
$$

where

$$
A=\left\{\begin{array}{c}
t+1, \text { if }-1 \leq t \leq 0 \\
1-t, \text { if } 0 \leq t \leq 1 \\
0, \text { otherwise }
\end{array}\right.
$$


and

$$
B=\left\{\begin{array}{c}
\frac{t+2}{2}, \text { if }-2 \leq t \leq 0 \\
\frac{2-t}{2}, \text { if } 0 \leq t \leq 2 \\
0, \text { otherwise }
\end{array}\right.
$$

Now consider the re-arrangement $Y$ of the sequence $X$

$$
Y=\{A, A, B, B, A, A, B, B, \ldots\} \notin w^{F}(\lambda) .
$$

But $X \in w^{F}(\lambda)$. This completes the proof.

Theorem 3.8. $w^{F}(\lambda)$ is not a convergence free space in general.

Proof. We consider the sequences

$$
\begin{gathered}
X_{k}(t)=\left\{\begin{array}{c}
k t+1, \text { if }-\frac{1}{k} \leq t \leq 0 \\
1-k t, \text { if } 0 \leq t \leq \frac{1}{k} \\
0, \text { otherwise }
\end{array}\right. \\
X_{k+1}(t)=\left\{\begin{array}{c}
(k+1) t+1, \text { if }-\frac{1}{k+1} \leq t \leq 0 \\
1-(k+1) t, \text { if } 0 \leq t \leq \frac{1}{k+1} \\
0, \text { otherwise }
\end{array}\right.
\end{gathered}
$$

Then

$$
\Delta X_{k}(t)=\left\{\begin{array}{c}
\frac{t\left(k^{2}+k\right)+2 k+1}{2 k+1}, \text { if }-\frac{1}{k}-\frac{1}{k+1} \leq t \leq 0 \\
\frac{(2 k+1)-t\left(k^{2}+k\right)}{2 k+1}, \text { if } 0 \leq t \leq \frac{1}{k}+\frac{1}{k+1} \\
0, \text { otherwise }
\end{array}\right.
$$

Now $\lim _{k \rightarrow \infty} \Delta X_{k}(t)=0$. Thus $\left(X_{k}\right) \in w^{F}(\lambda)$.

Again let

$$
\begin{gathered}
Y_{k}(t)=\left\{\begin{array}{c}
\frac{t+k}{k}, \text { if }-k \leq t \leq 0 \\
\frac{k-t}{k}, \text { if } 0 \leq t \leq k \\
0, \text { otherwise }
\end{array}\right. \\
Y_{k+1}(t)=\left\{\begin{array}{c}
\frac{t+(k+1)}{k+1}, \text { if }-(k+1) \leq t \leq 0 \\
\frac{(k+1)-t}{k+1}, \text { if } 0 \leq t \leq(k+1) \\
0, \text { otherwise }
\end{array}\right.
\end{gathered}
$$

Then

$$
\Delta Y_{k}(t)=\left\{\begin{array}{c}
\frac{t+(2 k+1)}{2 k+1}, \text { if }-(2 k+1) \leq t \leq 0 \\
\frac{(2 k+1)-t}{2 k+1}, \text { if } 0 \leq t \leq(2 k+1) \\
0, \text { otherwise }
\end{array}\right.
$$

Clearly $\left(Y_{k}\right) \notin w^{F}(\lambda)$. This completes the proof. 


\section{References}

[1] A. Esi, " $\lambda$-sequence spaces of interval numbers", Applied mathematics \& information sciences, vol. 8, no. 3, pp. 1099-1102, 2014, doi: $10.12785 /$ amis/ 080320

[2] A. H. Ganie and N. A. Sheikh, "Generalized difference sequence spaces of fuzzy numbers", New York journal of mathematics, vol. 19, pp. 431-438, 2013. [Online]. Available: https:/ / bit.ly/ 3wVSGZ]

[3] D. Burton and J. Coleman, "Quasi-Cauchy sequences", The American mathematical monthly, vol. 117, no. 4, pp. 328-333, 2010, doi: 10.4169/000298910X480793

[4] H. Çakall, "Forward continuity", Journal of computational analysis and applications, vol. 13, no. 2, pp. 225-230, 2011.

[5] H. Çakall,, "Statistical ward continuity", Applied mathematics letters, vol. 24, no. 10, pp. 1724-1728, 2011, doi: 10.1016/j.aml.2011.04.029

[6] H. Çakallı and B. Hazarika, "Ideal quasi-Cauchy sequences", Journal of inequalities and applications, vol. 2012, Art. ID. 234, doi: 10.1186/ 1029-242X-2012-234

[7] H. Çakall, "Statistical ward continuity", Applied mathematics letters, vol. 24, no. 10, pp. 1724-1728, 2011, doi: 10.1016/j.aml.2011.04.029

[8] H. Çakalll, "ס-quasi-Cauchy sequences", Mathematical and computer modelling, vol. 53, no. 1-2, pp. 397-401, 2011, doi: 10.1016/j.mcm. 2010.09.010

[9] H. Çakalli, I. Çanak, and M. Dik, " $\Delta$-quasi-slowly oscillating continuity", Mathematics and computation, vol. 216, no. 10, pp. 2865-2868, 2010, doi: 10.1016/j.amc.2010.03.137

[10] H. Fast, "Sur la convergence statistique", Colloquium mathematicae, vol. 2, no. 3-4, pp. 241-244, 1951. [Online]. Available: https:// bit.ly/ 3iwZo3f

[11] B. Hazarika, "On fuzzy real valued generalized difference I-convergent sequence spaces defined by Musielak-Orlicz function", Journal of intelligent \& fuzzy systems, vol. 25, no. 1, pp. 9-15, 2013, doi: 10.3233/ IFS-2012-0609

[12] B. Hazarika, "Lacunary difference ideal convergent sequence spaces of fuzzy numbers", Journal of intelligent \& fuzzy systems, vol. 25, no. 1, pp. 157-166, 2013, doi:10.3233/ IFS-2012-0622

[13] B. Hazarika, "On $\sigma$-uniform density and ideal convergent sequences of fuzzy real numbers", Journal of intelligent \& fuzzy systems, vol. 26, no. 2, pp. 793-799, 2014, doi: 10.3233/ IFS-130769 
[14] B. Hazarika and E. Savas, " $\lambda$-statistical convergence in n-normed spaces", Analele Universităţii "Ovidius" Constanţa. Seria Matematică (Online), vol. 21, no. 2, pp. 141-153, 2013, doi: 10.2478/auom2013-0028

[15] I. J. Schoenberg, "The integrability of certain functions and related summability methods" The American mathematical monthly, vol. 66, no. 5, pp. 361-375, 1959, doi: 10.1080/ 00029890.1959.11989303

[16] M. Mursaleen, " $\lambda$-statistical convergence", Mathematica slovaca, vol. 50, no. 1, pp. 111-115, 2000. [Online]. Available: https://bitly/ $2 \mathrm{~V} 68 \mathrm{hbN}$

[17] M. Matloka, "Sequences of fuzzy numbers", BUSEFAL, vol. 28, pp. 2837, 1986. [Online]. Available: https:/ / bit.ly/ 3zlQRXv

[18] B. C. Tripathy, "On generalized difference paranormed statistically convergent sequences", Indian journal of pure and applied mathematics, vol. 35, no. 5, pp. 655-663, 2004.

[19] B. C. Tripathy and M. Sen, "On generalized statistically convergent sequences", Indian journal of pure and applied mathematics, vol. 32, no. 11, pp. 1689-1694, 2001.

[20] B. C. Tripathy and A. Esi, "A new type of difference sequence spaces", International journal of science \& technology, vol. 1, no. 1, pp. 11-14, 2006. [Online]. Available: https:/ / bit.ly/ 3BoFakZ

[21] B. C. Tripathy and A. Baruah, "New type of difference sequence space of fuzzy real numbers", Mathematical modelling and analysis, vol. 14, no. 3, pp. 391-397, 2009, doi: 10.3846/ 1392-6292.2009.14.391-397

[22] B. C. Tripathy and P.C. Das, "Statistically convergent sequences of fuzzy real numbers", The journal of fuzzy mathematics, vol. 17, no. 1, pp. 119-129, 2009.

[23] B. C. Tripathy and P.C. Das, "Some classes of difference sequences of fuzzy real numbers", Fasciculi mathematici, vol. 40, pp. 105-117, 2008.

[24] L. A. Zadeh, "Fuzzy sets", Information and control, vol. 8, no. 3, pp. 338-353, 1965, doi: 10.1016/ S0019-9958(65)90241-X 